\title{
Host-cell sensors for Plasmodium activate innate immunity against liver-stage infection
}

\author{
Peter Liehl ${ }^{1}$, Vanessa Zuzarte-Luís ${ }^{1}$, Jennie Chan ${ }^{2,10}$, Thomas Zillinger ${ }^{3,10}$, Fernanda Baptista ${ }^{1}$, Daniel Carapau ${ }^{1}$, \\ Madlen Konert ${ }^{1}$, Kirsten K Hanson ${ }^{1}$, Céline Carret ${ }^{1}$, Caroline Lassnig ${ }^{4}$, Mathias Müller ${ }^{4}$, Ulrich Kalinke ${ }^{5}$, \\ Mohsan Saeed ${ }^{6}$, Angelo Ferreira Chora ${ }^{1}$, Douglas T Golenbock ${ }^{2}$, Birgit Strobl ${ }^{4}$, Miguel Prudêncio ${ }^{1}$, \\ Luis P Coelho ${ }^{1}$, Stefan H Kappe ${ }^{7}$, Giulio Superti-Furga ${ }^{8}$, Andreas Pichlmair ${ }^{8}$, Ana M Vigário ${ }^{1,9}$, Charles M Rice ${ }^{6}$, \\ Katherine A Fitzgerald ${ }^{2}$, Winfried Barchet $^{3} \&$ Maria M Mota ${ }^{1}$
}

\begin{abstract}
Before they infect red blood cells and cause malaria, Plasmodium parasites undergo an obligate and clinically silent expansion phase in the liver that is supposedly undetected by the host. Here, we demonstrate the engagement of a type I interferon (IFN) response during Plasmodium replication in the liver. We identified Plasmodium RNA as a previously unrecognized pathogenassociated molecular pattern (PAMP) capable of activating a type I IFN response via the cytosolic pattern recognition receptor Mda5. This response, initiated by liver-resident cells through the adaptor molecule for cytosolic RNA sensors, Mavs, and the transcription factors Irf 3 and Irf7, is propagated by hepatocytes in an interferon- $\alpha / \beta$ receptor-dependent manner. This signaling pathway is critical for immune cell-mediated host resistance to liver-stage Plasmodium infection, which we find can be primed with other PAMPs, including hepatitis C virus RNA. Together, our results show that the liver has sensor mechanisms for Plasmodium that mediate a functional antiparasite response driven by type I IFN.
\end{abstract}

Despite renewed eradication efforts by the international community, malaria still exerts an enormous disease burden, with nearly half the planet's population at risk for infection. There is a general consensus that the development of new intervention strategies is hampered by the current limited understanding of the biology of Plasmodium species and of the complex relationships that the parasite maintains with its hosts ${ }^{1}$. Malaria infection is initiated when sporozoites, injected by a female Anopheles mosquito, travel to the liver and infect hepatocytes, ultimately producing thousands of merozoites that will subsequently infect erythrocytes ${ }^{2}$. Whereas the liver stage of infection is clinically silent, erythrocyte infection leads to malaria and all its complications $^{2,3}$. This symptomatically silent feature of Plasmodium liver-stage infection, in such an immunoprivileged organ as the liver ${ }^{4}$, led to the widespread assumption that Plasmodium can infect and replicate inside hepatocytes undetected ${ }^{2,5}$. Nonetheless, we and others have reported the presence of inflammatory cells in the livers of infected mice ${ }^{6-9}$. Moreover, we have shown that in the absence of the host anti-inflammatory molecule heme oxygenase-1, liver-stage infection is impaired owing to a strong inflammatory response ${ }^{6}$. These findings suggest that the host senses Plasmodium hepatocyte infection and responds to it. However, the mechanisms used by the host to detect Plasmodium and mount an inflammatory response remain completely unknown ${ }^{5}$.

\section{RESULTS}

Plasmodium liver-stage infection induces a type I IFN response A comparison of a recently generated liver transcriptome ${ }^{10}$ between Plasmodium berghei sporozoite-infected mice and mock-infected mice (mice injected with an equivalent amount of noninfected salivary gland extract) revealed a statistically significant difference in the expression of 1,088 transcripts $(P<0.05$, Fig. 1a and Supplementary Table 1). Of those, 89 transcripts were at least twofold induced $(P<0.002$, Fig. $\mathbf{1 b}$ and Supplementary Table $\mathbf{1})$. Remarkably, all 89 genes were interferon-stimulated genes (ISGs) linked to the type I IFN signaling pathway ${ }^{11}$ (Supplementary Table 1). One main feature of the type I IFN response is the production and secretion of IFN- $\alpha$ and IFN- $\beta$, which signal in an autocrine or paracrine manner via the single interferon $\alpha$ receptor (IFNAR) composed of IFNAR1 and IFNAR2, ultimately inducing the expression of ISGs, known to mediate functional antiviral responses ${ }^{12}$. To confirm the activation of a host type I IFN response during Plasmodium liver infection, we analyzed by microarrays the expression of ISGs in mice lacking Ifnar 1

\footnotetext{
${ }^{1}$ Instituto de Medicina Molecular, Faculdade de Medicina Universidade de Lisboa, Lisboa, Portugal. ${ }^{2}$ Department of Medicine, Division of Infectious Diseases and Immunology, University of Massachusetts Medical School, Worcester, Massachusetts, USA. ${ }^{3}$ Institute of Clinical Chemistry and Clinical Pharmacology, University Hospital of Bonn, Bonn, Germany. ${ }^{4}$ Institute of Animal Breeding and Genetics and Biomodels, Austria University of Veterinary Medicine Vienna, Vienna, Austria. 5 Institute for Experimental Infection Research, TWINCORE, Centre for Experimental and Clinical Infection Research, Hannover Medical School and Helmholtz Centre for Infection Research, Hannover, Germany. ${ }^{6}$ Laboratory of Virology and Infectious Diseases, Center for the Study of Hepatitis C, The Rockefeller University, New York, New York, USA. ${ }^{7}$ Seattle Biomedical Research Institute, Seattle, Washington, USA. ${ }^{8}$ CeMM Research Center for Molecular Medicine of the Austrian Academy of Sciences, Vienna, Austria. ${ }^{9}$ Unidade de Ciências Médicas, Centro de compentência de ciências da vida, Universidade da Madeira, Funchal, Portugal. ${ }^{10}$ These authors contributed equally to this work. Correspondence should be addressed to M.M.M. (mmota@fm.ul.pt).
} 
a

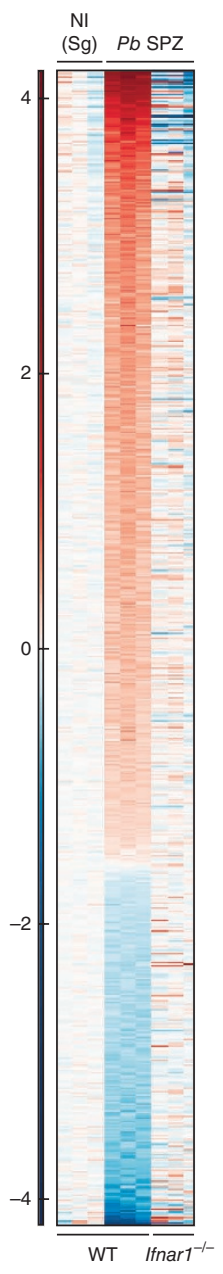

b

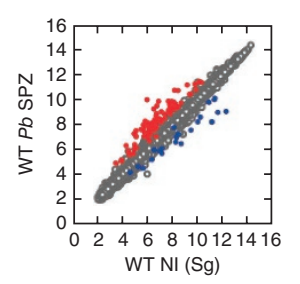

d

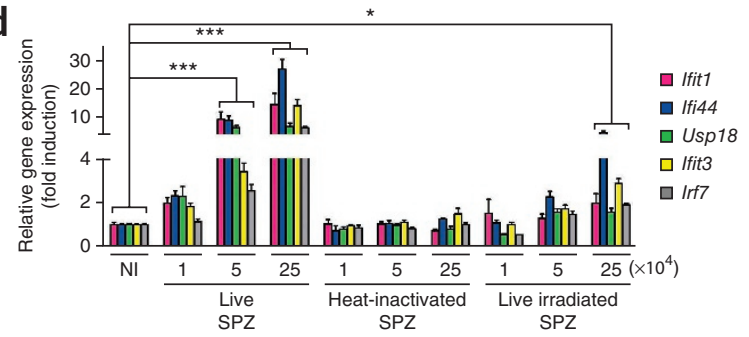

f

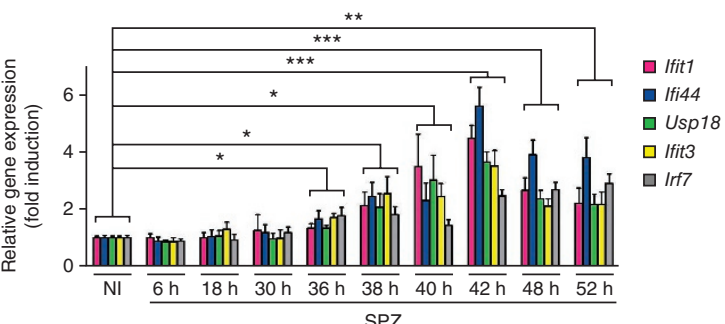

C
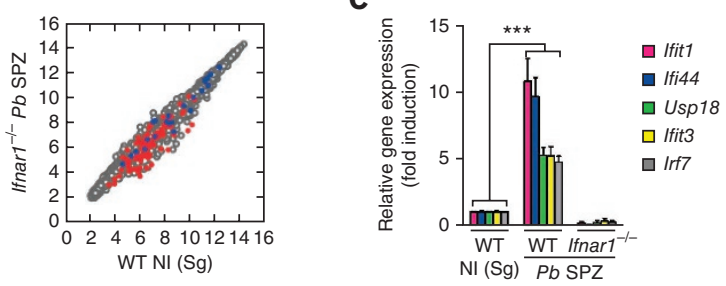

e

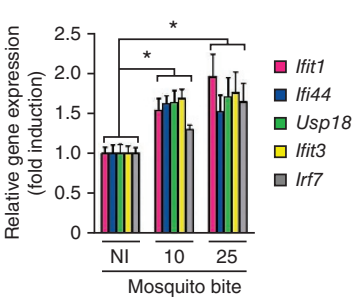

h
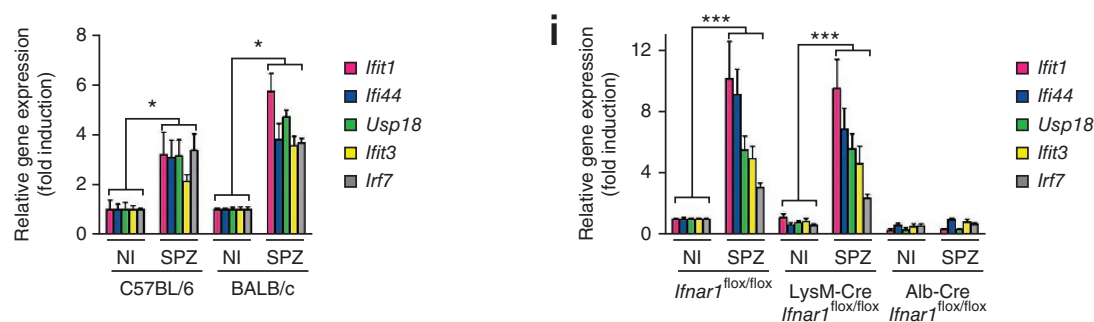

Figure 1 Plasmodium liver-stage infection induces a type I IFN response. (a) Heatmap showing differentially expressed transcripts in livers of WT and Ifnar $1^{-1-}$ mice $40 \mathrm{~h}$ after infection with $5 \times 10^{4} \mathrm{P}$. berghei ANKA $(P b)$ sporozoites (SPZ). Noninfected control mice were injected with an equivalent amount of noninfected salivary gland extract (NI (Sg)). Columns represent individual mice, and rows represent differentially expressed genes in $P$. berghei-infected WT mice compared to $\mathrm{NI}$ animals $(P<0.05)$. (b) Mean gene expression values in mice after infection with $P$. berghei sporozoites and in $\mathrm{NI}(\mathrm{Sg})$ control mice. Color identifies genes with more than twofold variation in WT infected mice $(P<0.05)$. Red: induced genes; Blue: repressed genes. (c) Expression profile of 5 representative ISGs in livers of WT and Ifnar 1-1- mice $42 \mathrm{~h}$ after infection. (d,e) ISG expression in livers of WT mice $42 \mathrm{~h}$ after infection by injection with the indicated numbers of live, heat-inactivated or irradiated $P$. berghei sporozoites (d) or by mosquito bite with 10 or 25 P. berghei-infected mosquitoes per mouse (e). (f) ISG expression in livers of WT mice at multiple time points after infection with P. berghei. (g,h) ISG expression in the livers of WT and Ifnar 1-/- mice infected with P. yoelii 17XNL (Py) sporozoites (g) and C57BL/6 and BALB/c mice

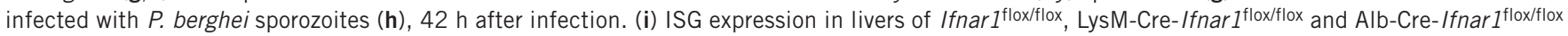
mice after infection with $P$. berghei sporozoites. The least significant $P$ value is shown, ${ }^{*} P<0.05 ;{ }^{* *} P<0.01 ;{ }^{* *} P<0.001$. Complete statistics in Supplementary Table 2. Data are expressed as means \pm s.e.m.

(Ifnar $1^{-/-}$mice), using our previously published wild-type data ${ }^{10}$ as a control. None of the genes differentially expressed in C57BL/6 wild-type (WT) mice in response to $P$. berghei infection were upregulated in the Ifnar $1^{-/-}$mice (Fig. 1a,b and Supplementary Table 1). We validated these differences by measuring the expression of several ISGs at the transcript (Ifit1, Ifi44, Usp18, Ifit3 and Irf7; used throughout as a readout for type I IFN pathway activation) and protein (Ifit1 and Dai) levels by quantitative RT-PCR (qRT-PCR) and western blot analysis, respectively (Fig. 1c and Supplementary Fig. 1). Induction of the type I IFN response was further visualized in liver slices of infected mice expressing lac $Z$ under the control of the promoter of Ifit1 (which encodes interferon-induced protein with tetratricopeptide repeats 1$)^{13}$ (Supplementary Fig. 1). The magnitude of the response upon infection was dose dependent
(Fig. 1d) and detectable after initiation of infection by mosquito bite (Fig. 1e and Supplementary Fig. 1). Time-course analysis first indicated significant ISG induction $36 \mathrm{~h}$ after infection, during the parasite replication phase. ISG induction peaked at $42 \mathrm{~h}$, still before the release of hepatic merozoites into the blood (Fig. 1f and Supplementary Fig. 1). Notably, we have shown that the IFN response is undetectable or impaired in mice infected with parasites that arrest before extensive replication ${ }^{14-16}$, with degree of response correlating with stage of parasite arrest; heat-inactivated sporozoites, which cannot invade hepatocytes, are completely unable to evoke a response (Fig. 1d and Supplementary Fig. 1). Another Plasmodium species able to infect rodents (Plasmodium yoelii) was also able to induce a type I IFN response in WT mice but not in Ifnar $1^{-1-}$ mice (Fig. 1g). Furthermore, P. berghei induced a similar 
a

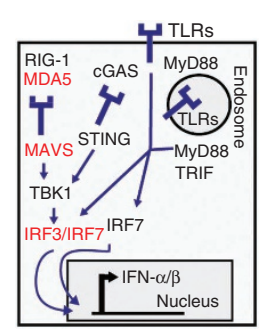

b $\square$ Ifit1 $\square$ Ifi44 $\square$ Usp18 $\square$ Ifit3 $\square$ Irf7

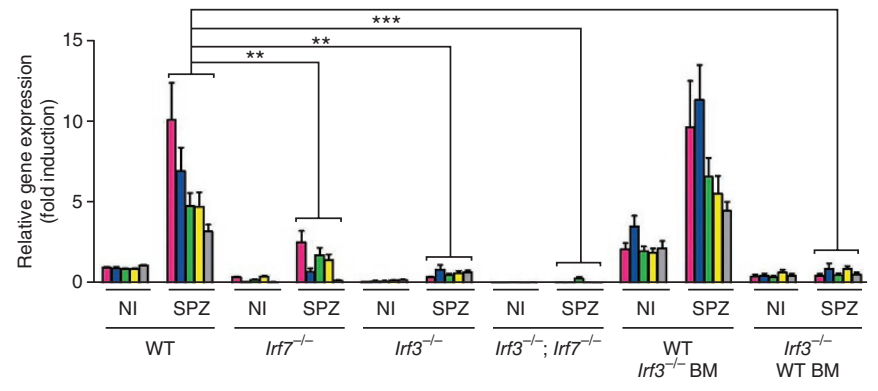

C $\quad$ Ifit1 $\square$ Ifi44 $\square$ Usp18 $\square$ Ifit3 $\square$ Irf7

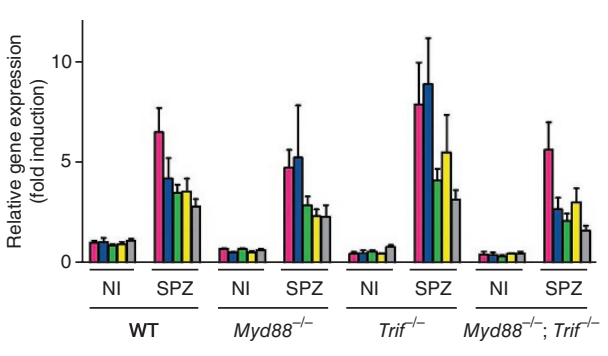

d $\quad$ Iffit1 $\square$ Ifi44 $\square$ Usp18 $\square$ Ifit3 $\square$ Irf7

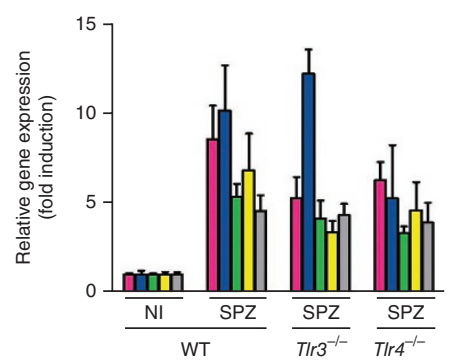

e $\quad$ Ifit1 $\square$ Ifi44 $\square$ Usp18

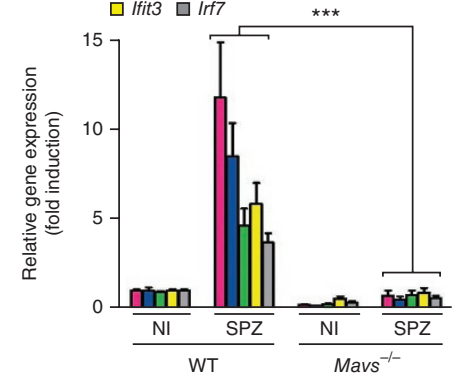

f $\quad$ Ifit1 $\square$ lfi44 $\square$ Usp18 $\square$ Ifit3 $\square$ Irf7

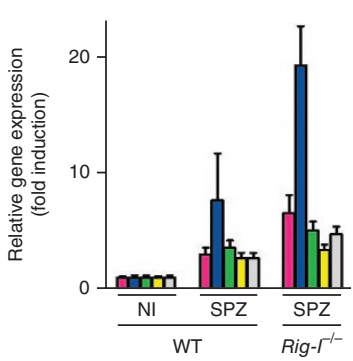

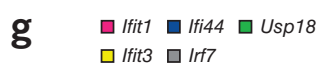

$$
\square \text { Ifit3 } \square \text { Irf7 }
$$

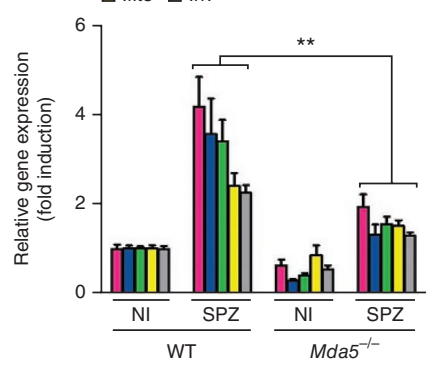

Figure 2 Plasmodium-mediated type I IFN induction requires Mavs, and Mda5 senses Plasmodium RNA. (a) Schematic representation of type I IFN signaling pathways leading to the induction of IFN- $\alpha$ and IFN- $\beta$ (IFN- $\alpha / \beta)$ transcription. cGAS, cyclic GMP-AMP synthase. (b) Gene expression analysis of 5 representative ISGs in livers of WT, $I r f 7^{-1-}, I r f 3^{-1-}$ and Irf $7^{-1-}$; Irf3 ${ }^{-1-}$ mice as well as bone marrow (BM)-chimeric mice (WT mice with Irf3-l- BM and $/ \mathrm{rf3}^{-/-}$mice with WT BM) $42 \mathrm{~h}$ after infection with $5 \times 10^{4} \mathrm{P}$. berghei sporozoites.

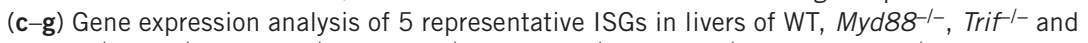

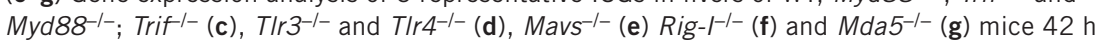
after infection with $5 \times 10^{4} P$. berghei sporozoites. Statistical analysis was performed for each individual gene; the least significant $P$ value is shown, ${ }^{*} P<0.05$. (h) ISG expression in livers of WT and $\mathrm{Mda5}^{-1-}$ mice $4 \mathrm{~h}$ after hydrodynamic injection with $50 \mu \mathrm{g}$ per mouse of $P$. berghei

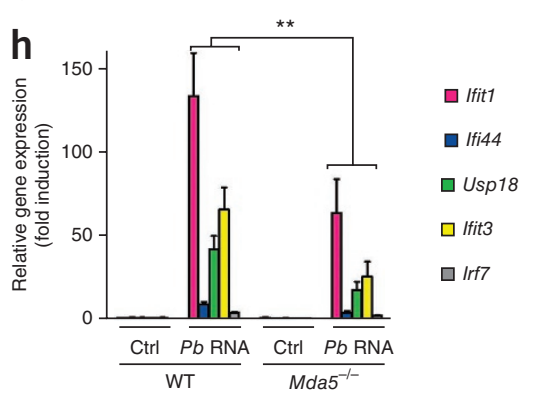
RNA. Expression of individual genes from sporozoite-infected livers was tested against mock-injected control samples. The least significant $P$ value of all samples is shown, ${ }^{* *} P<0.01$. Complete statistics in Supplementary Table 2. Data are expressed as means \pm s.e.m.

response in WT BALB/c mice, as well (Fig. 1h), which indicates that induction of a type I IFN response is restricted to neither Plasmodium species nor mouse host strain.

The fact that the P. berghei-induced type I IFN response is detectable in whole-liver extracts even after physiological mosquito bite infection with few sporozoites (leading to a small ratio of infected over uninfected hepatocytes) strongly suggests a mechanism of amplification of the signaling, as usually described for the type I IFN response. Thus, we next investigated which cell types in the liver are required for the propagation of the type I IFN response. P. berghei-infected mice lacking Ifnar1 specifically on myeloid cells including macro-

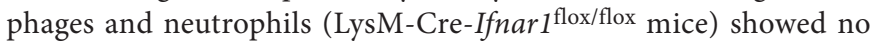
reduction in the induction of ISGs compared to the WT mice, whereas the response was completely impaired in mutant mice lacking Ifnar1

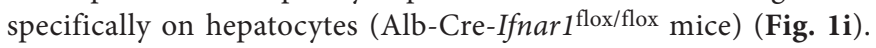
Altogether, to our knowledge, our data reveal for the first time that Plasmodium liver-stage infection induces a typical type I IFN signature in the liver of infected mice, with hepatocytes being the primary source of the ISG expression.

\section{Plasmodium RNA induces type I IFN through Mda5 and Mavs}

Having demonstrated that Plasmodium liver-stage infection specifically induces a typical type I IFN signature in the livers of infected mice, we next sought to identify the molecules involved in this induction.
Regardless of which pattern recognition receptor (PRR) initiates the type I IFN response, all the signaling pathways described so far converge upon two downstream transcription factors, interferon regulatory factor (IRF3) and IRF7, which regulate the transcription of type I IFNs ${ }^{17}$ (Fig. 2a). As expected, the type I IFN response was significantly impaired in $\mathrm{Irf3} 3^{-/-}$and $\mathrm{Irf7} 7^{-/-}$mice, as well as in $\mathrm{Irf3} 3^{-/-}$; $I r f 7^{-1-}$ mice, demonstrating that both Irf3 and Irf7 are critical mediators of the host type I IFN response to $P$. berghei liver-stage infection (Fig. 2b). We then established $\operatorname{Irf3} 3^{-/-}$bone marrow-chimeric mice to address the respective roles of liver-resident cells (such as hepatocytes) and circulating immune cells (such as neutrophils) in initiating the $P$. berghe $i$-induced type I IFN response. Whereas Irf3 expression was dispensable in circulating immune cells, it was required in liverresident cells to induce the type I IFN response during $P$. berghei liver-stage infection (Fig. 2b), demonstrating that recognition of a PAMP from $P$. berghei occurs in a liver-resident cell.

We next sought to identify the PRR pathway responsible for the sensing event initiating the type I IFN response. Type I IFN production can be triggered by various PRRs, such as transmembrane Toll-like receptors (including TLR2, TLR3, TLR4, TLR5, TLR7 and TLR9) ${ }^{18,19}$ and cytosolic RNA and DNA sensors. As all TLRs signal through at least one of two possible adaptors, myeloid differentiation factor 88 (MyD88) and TIR-domain-containing adapter-inducing interferon- $\beta$ (TRIF), we next tested their function in the Plasmodium-induced 
a

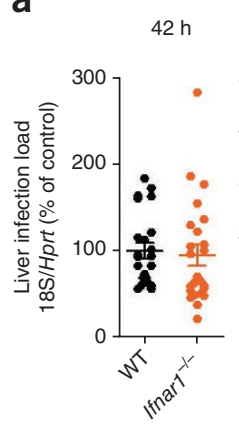

b

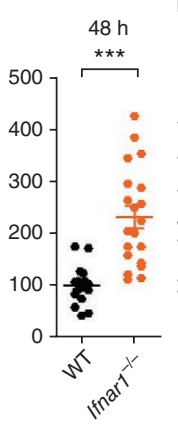

b $\quad c$

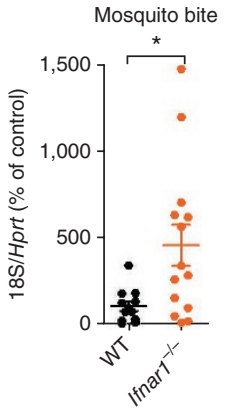

c

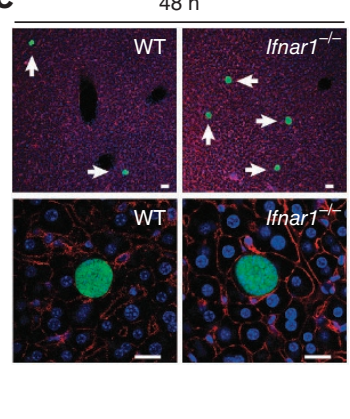

d

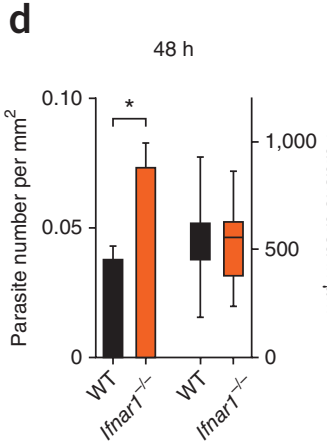

e

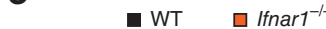

Figure 3 The type I IFN response is required for in vivo host defense. (a) Parasite liver load in WT and Ifnar $1^{-1-}$ mice measured by qRT-PCR of $P$. berghei $18 \mathrm{~S} \mathrm{rRNA}, 42 \mathrm{~h}$ and $48 \mathrm{~h}$ after infection normalized to hypoxanthine-guanine phosphoribosyltransferase (Hprt) and plotted as percentage of the levels in WT mice. (b) Parasite liver load in WT and Ifnar $1^{-/-}$mice $50 \mathrm{~h}$ after infection by mosquito bite using 10 mosquitoes per mouse and plotted f
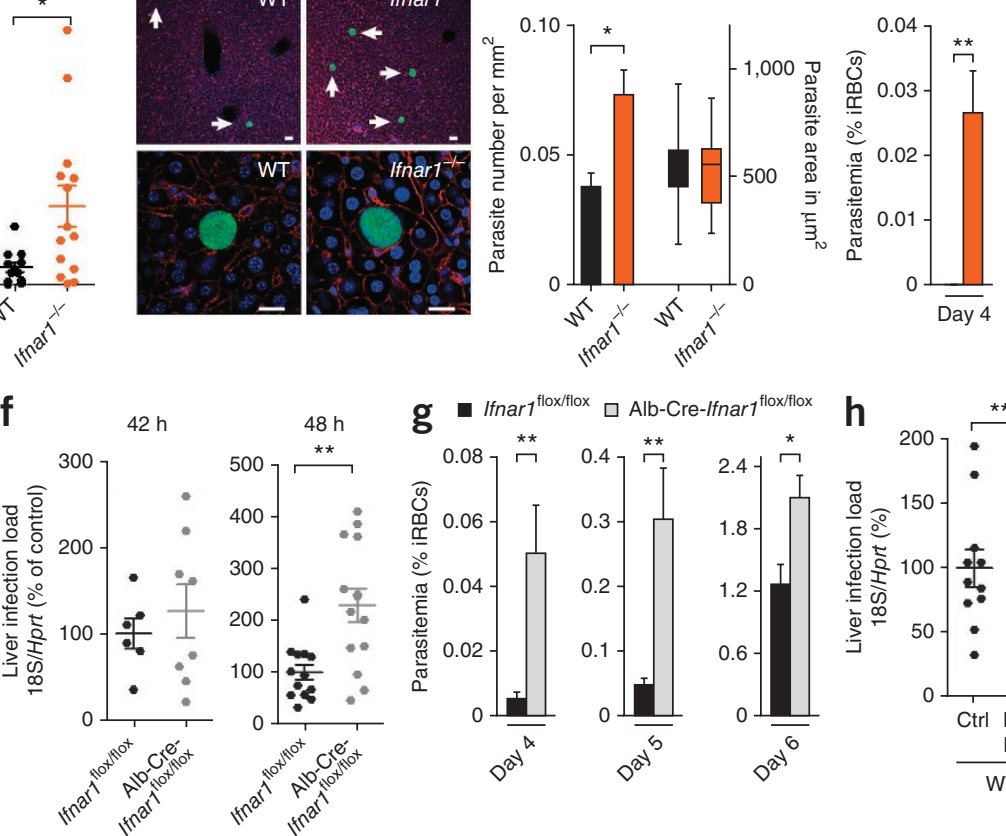

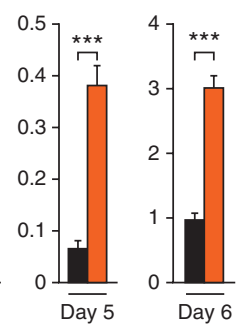
as percentage of the levels in WT mice.

(c) Representative fluorescence images of liver sections of WT and Ifnar $1^{-/-}$mice, $48 \mathrm{~h}$ after infection with GFP-expressing $P$. berghei sporozoites; parasite in green, DNA stained with DAPI (blue) and F-actin with phalloidin Alexa555 (red); arrows indicate parasite EEFs. Top scale bars, $40 \mu \mathrm{m}$; bottom scale bars, $20 \mu \mathrm{m}$. (d) EEF size (area) and density (number) in WT and Ifnar ${ }^{-l-}$ mice, analyzed by microscopy $48 \mathrm{~h}$ after infection with P. berghei sporozoites. (e) Parasitemia in WT and Ifnar $1^{-/}$mice after infection with 500 sporozoites. iRBCs, infected red blood cells. (f) Parasite liver load in Ifnar $\mathrm{flox}^{\mathrm{fl} / \mathrm{lox}}$ and Alb-Cre-Ifnar $\mathrm{I}_{\text {flox/flox }}$ mice, as measured by qRT-PCR, $42 \mathrm{~h}$ and $48 \mathrm{~h}$ after infection with $5 \times 10^{4} \mathrm{P}$. berghei sporozoites and

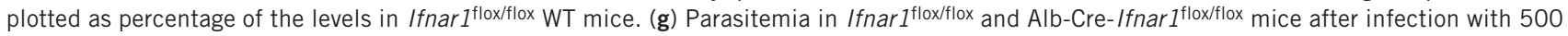
P. berghei sporozoites. (h) Parasite liver load in WT and Ifnar $1^{-1-}$ mice treated with HCV RNA ( $50 \mu \mathrm{g}$ per mouse, $20 \mathrm{~h}$ before infection), as measured by qRT-PCR, $42 \mathrm{~h}$ after infection with $P$. berghei sporozoites. Complete statistics in Supplementary Table 2. Data are expressed as means \pm s.e.m. ${ }^{*} P<0.05 ;{ }^{*} P<0.01 ;{ }^{* *} P<0.001$.

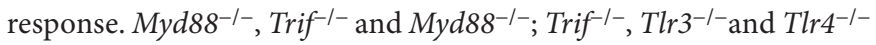
mice were all capable of responding to $P$. berghei infection (Fig. $\mathbf{2 c}, \mathbf{d})$, which suggests that TLRs are not the sensors responsible for driving the type I IFN response during liver-stage infection. An alternative pathway of type I IFN induction proceeds through the mitochondrial antiviral signaling protein (MAVS; also known as CARDIF, VISA and IPS1) ${ }^{20-23}$. Mavs $^{-1-}$ mice were not able to induce the expression of ISGs after $P$. berghei liver-stage infection (Fig. 2e), showing that Mavs is a key molecule in the induction of the type I IFN response during P. berghei infection. Collectively, these data show that the Plasmodiummediated induction of the type I IFN response requires signaling through Mavs and is independent of TLR signaling.

Two known cytoplasmic PRRs of the family of retinoic acidinducible gene-I (RIG-I)-like receptors (RLRs), RIG-I and melanoma differentiation-associated gene 5 (MDA5), are known to trigger a MAVS-dependent type I IFN response. Although both RIG-I and MDA5 have been shown to sense viral RNA ${ }^{24-26}$, it is not known whether either is able to sense RNA from protozoan parasites. Thus, we assessed whether Plasmodium RNA could be a PAMP capable of eliciting a type I IFN response through either RIG-I or MDA5. Transfection of RNA from various Plasmodium species (P. berghei, $P$. yoelii and $P$. falciparum) into bone marrow-derived dendritic cells or mouse WT, Mda5 $5^{-/-}$and $D d x 58^{-/-}$(here called Rig-I $^{-/-}$) primary hepatocytes led to the production of type I IFNs in a Rig-I-independent but Mda5-dependent manner (Supplementary Fig. 2). Still, we examined the ability of both $\mathrm{Rig}^{-\mathrm{I}^{-/-}}$and $\mathrm{Mda5^{-/- }}$ mice to mount a type I IFN response after Plasmodium infection. Consistent with our results from Plasmodium RNA transfection experiments, the type I IFN response was significantly impaired in infected $M d a 5^{-/-}$but not Rig-I ${ }^{-1-}$ mice compared to WT controls (Fig. 2f,g). Hydrodynamic tail vein injection of purified $P$. berghei RNA in vivo also induced a partial Mda5-dependent type I IFN response (Fig. 2h). Overall, our data show that Plasmodium RNA is a PAMP recognized by Mda5 but not Rig-I and that during infection, liver-resident cells sense Plasmodium parasites via Mda5 and yet unknown other receptors.

\section{The type I IFN response is required for in vivo host defense}

Type I IFN responses are known to be critical for host defense against viral infections, but their efficacy against bacterial and parasitic infections is still poorly defined 27,28 . In our model, Ifnar $1^{-/-}$and $\operatorname{Irf3} 3^{-/-}$ mice exhibited a significant increase in parasite liver load at $48 \mathrm{~h}$ after P. berghei sporozoite injection, or after mosquito bite infection with P. berghei-infected mosquitoes, compared to their WT counterparts (Fig. 3a,b and Supplementary Fig. 3). Microscopic examination of thick liver sections from WT and Ifnar $1^{-1-}$ mice $48 \mathrm{~h}$ after infection revealed that in the absence of type I IFN-dependent signaling, the number of liver-stage parasites was substantially increased (Fig. 3c,d). When we initiated infections even with low numbers (500) of $P$. berghei sporozoites, we detected parasites in the blood $1 \mathrm{~d}$ earlier in Ifnar $1^{-1-}$ mice than in WT mice, which is reflected in significant differences in blood-stage parasitemia (Fig. 3e). Furthermore, mice lacking Ifnar in hepatocytes only (Alb-Cre-Ifnar $1^{\text {flox/flox }}$ ) had a significant increase 
Figure 4 Liver leukocytes responding to type I IFN are recruited to infected hepatocytes and are critical in parasite elimination. (a) Parasite liver load in WT and Ifnar ${ }^{-1-}$ mice treated with DMXAA ( $500 \mu \mathrm{g}$ per mouse, $20 \mathrm{~h}$ before infection), as measured by qRT-PCR, $42 \mathrm{~h}$ after infection with $P$. berghei sporozoites and plotted as percentage of mock-treated mice. (b) Parasite load in primary hepatocytes treated with DMXAA (100 $\mu \mathrm{g} \mathrm{ml}^{-1}, 3 \mathrm{~h}$ before infection), as measured by qRT-PCR, $44 \mathrm{~h}$ after infection with $P$. berghei sporozoites and plotted as percentage of mock-treated cells. (c) Immunofluorescence staining of a liver section showing infected hepatocyte surrounded by immune cells $44 \mathrm{~h}$ after infection with $P$. berghei sporozoites. Parasite in green, DNA stained with DAPI (blue) and F-actin with phalloidin Alexa555 (red); arrow indicates infiltrating immune cells. Scale bar, $20 \mu \mathrm{m}$. (d) Percentage of EEFs associated with inflammatory foci in WT and Ifnar $1^{-/-}$mice $(n=3) 44,48$ and $52 \mathrm{~h}$ after infection. Statistically significant differences were calculated using the Chi-squared test. (e) Gene expression analysis of 5 representative

ISGs in isolated hepatocytes and liver leukocytes 42 and $48 \mathrm{~h}$ after infection with $P$. berghei sporozoites. The least significant $P$ value is shown. (f) Percentage of EEFs associated with inflammatory foci in Ifnar $1^{\text {flox/flox }}$ and LysM-Cre-Ifnar $1^{\text {floxflox }}$ mice $(n=3) 48$ and $52 \mathrm{~h}$ after infection. Statistically significant differences were calculated using the Chi-squared test. (g) Parasite liver load in Ifnar $1^{\text {flox/flox }}$ and LysM-Cre-Ifnar $1^{\text {flox/flox }}$ mice measured by qRT-PCR, 42 and $48 \mathrm{~h}$ after infection with $P$. berghei sporozoites and plotted as percentage of the levels in WT mice. Complete statistics in Supplementary Table 2. Data are expressed as means \pm s.e.m. ${ }^{*} P<0.05 ;{ }^{*} P<0.01 ;{ }^{* * *} P<0.001$.

in both parasite liver load and blood-stage parasitemia as compared to their WT counterparts (Fig. 3f,g), demonstrating that the differences in blood-stage parasitemia are mainly due to hepatocyte-mediated type I IFN signaling. This highlights the impact of liver-specific type I IFN on total parasite biomass during initiation of blood-stage infection, which influences malaria severity ${ }^{29}$.

We next sought to determine whether a functional antiplasmodial type I IFN response could be triggered by other hepatotropic pathogens, such as hepatitis $\mathrm{C}$ virus ( $\mathrm{HCV}$ ), which is highly prevalent in malaria-endemic areas ${ }^{30}$. Hydrodynamic tail vein injection of HCV RNA before P. berghei sporozoite infection led to a significant reduction in parasite liver load at $48 \mathrm{~h}$ after infection in an Ifnar1-dependent manner (Fig. 3h). Overall, we demonstrate that an innate immune antiparasitic response contributes to host resistance during Plasmodium liver-stage infection and raise the possibility of a role for type I IFN signaling in the interplay between hepatotropic infections in humans.

\section{Liver leukocytes are critical in parasite elimination}

Finally, we sought to gain insight into the effector mechanisms by which the type I IFN response increases host resistance to liver-stage Plasmodium parasites. During viral infections, ISGs can act as effectors directly mediating the type I IFN antiviral defense, with different viruses being targeted by unique sets of ISGs ${ }^{11}$. Exogenous induction of a type I IFN response by 5,6-dimethylxanthenone-4-acetic acid (DMXAA, a drug that activates Irf3 (refs. 31,32)) led to a significant Ifnar1-dependent reduction in liver-stage infection in vivo, but not in mouse primary hepatocytes ex vivo (Fig. 4a,b and Supplementary Fig. 4). This result suggests that the hepatocyte-mediated response is not able to eliminate the parasite and indicates that although hepatocytes are essential for the propagation of the type I IFN response, other cells may be required as effectors in parasite elimination.
The type I IFN response is known to activate and recruit immune cells to the site of bacterial infection ${ }^{33}$, and inflammatory cells have been noted to infiltrate the livers of Plasmodium-infected mice $6,7,9$. Thus, we next tested whether the presence of these inflammatory cells in the liver during Plasmodium infection could depend on the type I IFN response. We observed that the number of infected hepatocytes surrounded by infiltrates increases with time after the peak of type I IFN response in an Ifnar-dependent manner (Fig. 4c,d), consistent with a role for the type I IFN response in establishing these infiltrates around infected cells. We would therefore expect these infiltrating leukocytes to show a characteristic IFN response, and indeed, leukocytes purified from infected livers (including both infiltrating and resident cells) showed significant ISG induction at $48 \mathrm{~h}$, whereas the purified hepatocyte populations showed a peak at $42 \mathrm{~h}$ (Fig. 4e). Moreover, the number of infected hepatocytes surrounded by immune cells was significantly reduced in $P$. berghei-infected mice lacking Ifnar1 specifically on myeloid cells (LysM-Cre-Ifnar $1^{\text {flox/flox }}$ ), including macrophages and neutrophils (Fig. 4f). Thus, our data indicate that leukocytes capable of responding to type I IFN are mobilized to the vicinity of infected hepatocytes by hepatocyte-propagated type I IFN signaling. LysM-Cre-Ifnar $1^{\text {flox/flox }}$ mice also had a significantly higher parasite liver load compared to WT mice at 48 h, i.e., when leukocytes themselves were responding to type I IFN; consistent with our previous results, this effect on parasite load was not present at $42 \mathrm{~h}$, when infiltrates were not yet numerous and leukocytes lacked significant ISG induction (Fig. 4d-g). Overall, these data suggest that the ISG response induced in hepatocytes by Plasmodium liver infection is not able to eliminate Plasmodium parasites directly and that hepatocyte-driven propagation of the type I IFN signaling after the initial sensing events is critical for a later recruitment and induction of a type I IFN response in leukocytes, which probably act as effectors, playing a key role in parasite elimination (Fig. 5). 
Figure 5 Schematic representation of the proposed sequence of events occurring in the liver after Plasmodium infection. Infection of hepatocytes by Plasmodium parasites induces a type I IFN response, which is triggered in liver-resident cells by Plasmodium RNA via the cytoplasmic RNA sensor Mda5 and other unknown receptors, Mavs and the transcription factors Irf3 and Irf7 (1). Type I IFNs are then released in the extracellular environment (2). It binds to Ifnar on the surface of hepatocytes, propagating the response in an autocrine and paracrine manner (3). In turn, Ifnar activation leads to the upregulation of ISG transcription (including chemokines) and propagation of the response throughout the entire liver (4). IFN and the chemokines released from hepatocytes activate (5) and direct (6) innate immune cells to the infected hepatocytes, which have a key role in parasite elimination (7).

\section{DISCUSSION}

Here, we show that the liver possesses the molecular machinery capable of sensing Plasmodium RNA, a previously unrecognized PAMP, and that parasites are sensed during liver-stage infection, triggering a functional type I IFN response that reduces parasite burden. The initial signaling cascade upon sensing is triggered in liver-resident cells and depends on the cytoplasmic RNA sensor Mda5, the adaptor molecule Mavs and the transcription factors Irf3 and Irf7. We demonstrate that Mda5 is required to recognize Plasmodium RNA and responds by inducing type I IFN production, which constitutes what we believe is the first description of a nonviral ligand for Mda5. Interestingly, double-stranded noncoding Plasmodium RNAs have been described ${ }^{34}$. However, the observation that $M d a 5^{-/-}$mice are not as impaired as $\mathrm{Mavs}^{-/-}$mice in the type I IFN response suggests that other sensors or recognition mechanisms for Plasmodium exist (Fig. 5). Additionally, RNA may not be the only Plasmodium PAMP detected in the liver; non-nucleic acids may also contribute, as recently suggested for HIV-1 infection ${ }^{35}$. Our results suggest a model in which the activation of the cytosolic signaling pathways leads to the release of type I IFNs into the extracellular environment where it binds to Ifnar on the surface of hepatocytes, propagating the response in an autocrine and paracrine manner via the production of ISGs. We hypothesize that chemoattractant molecules released from type I IFN-producing hepatocytes activate and recruit leukocytes to the liver, where they would be further targeted specifically to infected hepatocytes (see Fig. 5). Future studies should decipher the exact mechanisms at work in this recruitment and the chemoattractant molecules involved.

Our work reveals that the host does not remain passive during the liver stage of infection. On the contrary, the host innate immune system has developed ways to recognize and control parasite load during the first obligatory step of Plasmodium infection in the mammalian host, as well as to reduce the number of parasites able to emerge from the liver and initiate the blood stage of infection. Although this innate immune response does not eliminate every parasite, which implies that the parasite has probably devised strategies to counteract it ${ }^{5,6}$, the observed reduction in surviving exoerythrocytic forms may be physiologically important on a population level, by decreasing parasite genetic diversity in the resulting blood-stage population. This is highly relevant knowledge for new drug and vaccine strategies, as the type I IFN response might reduce the likelihood of transmission of drug-resistant parasites or the escape of attenuated parasites used in vaccination. Further, using adjuvants (for liver-stagetargeted vaccines) or prophylactics that enhance the potency of the host's natural type I IFN response could lead to a better control of liver-stage parasites. Notably, our data demonstrate that preactivation of a type I IFN response impairs the establishment of Plasmodium

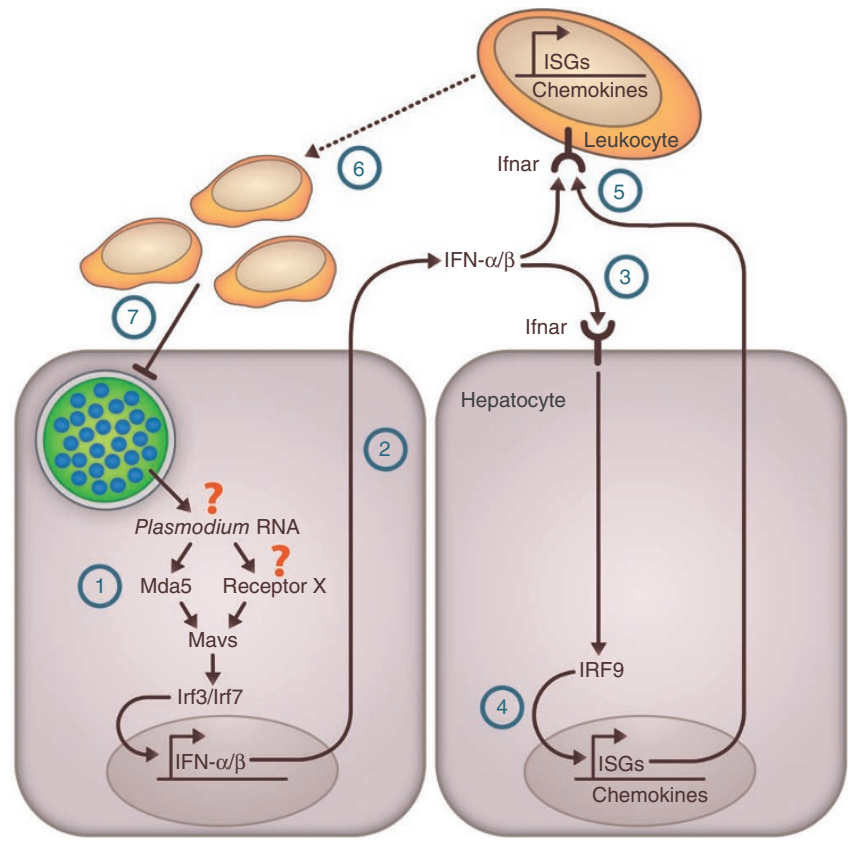

infection. This observation is clinically relevant for most populations exposed to malaria, as they not only face multiple re-infections with Plasmodium ${ }^{10}$ but also are commonly infected with hepatotropic pathogens such as $\mathrm{HCV}^{36,37}$, increasing the likelihood of interference between various pathogens and the immune response within a single host. These results highlight the fact that host-pathogen interactions can no longer be perceived as a dialog between a host and a single pathogen of interest, but rather as nodes of complex interactions modulated by the host's entire network of symbionts and pathogens. Undeniably, further investigation of both host recognition mechanisms and subversion strategies that the parasite has most certainly developed over million years of coevolution will be useful not only for malaria vaccine development but also for the sustained success of both malaria-control and Plasmodium-elimination agendas.

\section{METHODS}

Methods and any associated references are available in the online version of the paper.

Accession codes. Microarray data have been deposited in ArrayExpress with accession code E-MTAB-1145.

Note: Any Supplementary Information and Source Data files are available in the online version of the paper.

\section{ACKNOWLEDGMENTS}

$\mathrm{Mavs}^{-/-}$mice were from Z.J. Chen (South Western Medical School). Mda5 ${ }^{-/-}$

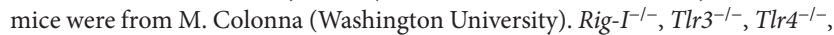
Trif $^{-1-}$ and $M y d 88^{-1-}$ mice were a gift from S. Akira (Osaka University). Trif ${ }^{-1-}$ and

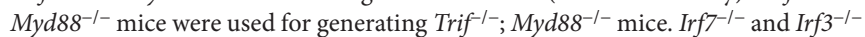
mice were kindly provided by T. Taniguchi (University of Tokyo). We would like to thank A. Parreira for Anopheles stephensi production and infection, M. Rebelo for help with $P$. falciparum in vitro cultures, J. Ribot for help with chimeric mice and A. Zaidman-Rémy for critical reading of the manuscript. We are grateful to M. Seabra (CEDOC, Faculdade de Ciências Médicas) and A. Holder (UK Medical Research Council National Institute for Medical Research) for providing antisera. This work was supported by Fundação para a Ciência e Tecnologia (FCT, Portugal) grants (PTDC/SAU-GMG/100313/2008 to M.M.M., PTDC/SAUGMG/104392/2008 to C.C. and PTDC/SAU-MIC/113697/2009 to V.Z.-L., and also by the European Research Council grant ERC-2012-StG_20111109 to M.M.M., the European Community's Seventh Framework Programme (FP7/2007-2013) 
grant agreement 242095 to M.M.M., US National Institutes of Health grant AI079293 to K.A.F. and D.T.G., Austrian Science Fund grant SFB F28 to M.M. and B.S., Deutsche Forschungs Gesellschaft grants BA3544/1-1 and SFB704 (to W.B.), the Starr Foundation, the Greenberg Medical Research Institute and Bill \& Melinda Gates Foundation grant OPP1021571 (subcontract R10291, M11R11017, all to C.M.R.). P.L. was supported by Fondation pour la Recherche Médicale and FCT (fellowship SFRH/BPD/41547/2007). V.Z.-L. was supported by European Molecular Biology Organization fellowship ALTF357-2009. D.C. and C.C. were supported by FCT fellowships (SFRH/BPD/64626/2009 and SFRH/BPD/40965/2007, respectively). M.S. was supported in part by a Helmsley Postdoctoral Fellowship for Basic and Translational Research on Disorders of the Digestive System at The Rockefeller University.

\section{AUTHOR CONTRIBUTIONS}

P.L., V.Z.-L., J.C., T.Z., F.B., D.C., M.K., K.K.H., B.S., A.F.C., M.P. and A.M.V. performed experiments. C.C. and L.P.C. performed the bioinformatic analysis. M.M., C.L., U.K., D.T.G., G.S.-F., A.P., K.A.F. and W.B. provided transgenic mice. M.S., C.M.R., G.S.-F. and A.P. provided essential reagents. S.H.K. provided expertise. P.L. and M.M.M. conceived the study and designed the experimental procedures. M.M.M. supervised the study. P.L. and M.M.M. wrote the manuscript. V.Z.-L., T.Z., K.K.H., M.S., K.A.F. and W.B. provided insightful comments to the manuscript. All authors read and approved the manuscript.

\section{COMPETING FINANCIAL INTERESTS}

The authors declare no competing financial interests.

Reprints and permissions information is available online at http://www.nature.com/ reprints/index.html.

1. malERA Consultative Group on Basic Science and Enabling Technologies. A research agenda for malaria eradication: basic science and enabling technologies. PLoS Med. 8, e1000399 (2011).

2. Prudêncio, M., Rodriguez, A. \& Mota, M.M. The silent path to thousands of merozoites: the Plasmodium liver stage. Nat. Rev. Microbiol. 4, 849-856 (2006).

3. Pamplona, A., Hanscheid, T., Epiphanio, S., Mota, M.M. \& Vigario, A.M. Cerebral malaria and the hemolysis/methemoglobin/heme hypothesis: shedding new light on an old disease. Int. J. Biochem. Cell Biol. 41, 711-716 (2009).

4. Lang, K.S. et al. Immunoprivileged status of the liver is controlled by Toll-like receptor 3 signaling. J. Clin. Invest. 116, 2456-2463 (2006).

5. Liehl, P. \& Mota, M.M. Innate recognition of malarial parasites by mammalian hosts. Int. J. Parasitol. 42, 557-566 (2012).

6. Epiphanio, S. et al. Heme oxygenase-1 is an anti-inflammatory host factor that promotes murine plasmodium liver infection. Cell Host Microbe 3, 331-338 (2008).

7. Khan, Z.M., Ng, C. \& Vanderberg, J.P. Early hepatic stages of Plasmodium berghei: release of circumsporozoite protein and host cellular inflammatory response. Infect. Immun. 60, 264-270 (1992).

8. Leiriao, P., Mota, M.M. \& Rodriguez, A. Apoptotic Plasmodium-infected hepatocytes provide antigens to liver dendritic cells. J. Infect. Dis. 191, 1576-1581 (2005).

9. van de Sand, C. et al. The liver stage of Plasmodium berghei inhibits host cell apoptosis. Mol. Microbiol. 58, 731-742 (2005).

10. Portugal, S. et al. Host-mediated regulation of superinfection in malaria. Nat. Med. 17, $732-737$ (2011)

11. Schoggins, J.W. et al. A diverse range of gene products are effectors of the type I interferon antiviral response. Nature 472, 481-485 (2011).
12. Decker, T., Muller, M. \& Stockinger, S. The yin and yang of type I interferon activity in bacterial infection. Nat. Rev. Immunol. 5, 675-687 (2005).

13. Pichlmair, A. et al. Ifit 1 is an antiviral protein that recognizes $5^{\prime}$-triphosphate RNA. Nat. Immunol. 12, 624-630 (2011).

14. Mueller, A.K. et al. Plasmodium liver stage developmental arrest by depletion of a protein at the parasite-host interface. Proc. Natl. Acad. Sci. USA 102, 3022-3027 (2005).

15. Mueller, A.K., Labaied, M., Kappe, S.H. \& Matuschewski, K. Genetically modified Plasmodium parasites as a protective experimental malaria vaccine. Nature 433, 164-167 (2005).

16. van Dijk, M.R. et al. Genetically attenuated, P36p-deficient malarial sporozoites induce protective immunity and apoptosis of infected liver cells. Proc. Natl. Acad. Sci. USA 102, 12194-12199 (2005).

17. Honda, K. \& Taniguchi, T. Irfs: master regulators of signalling by Toll-like receptors and cytosolic pattern-recognition receptors. Nat. Rev. Immunol. 6, 644-658 (2006).

18. Barbalat, R., Lau, L., Locksley, R.M. \& Barton, G.M. Toll-like receptor 2 on inflammatory monocytes induces type I interferon in response to viral but not bacterial ligands. Nat. Immunol. 10, 1200-1207 (2009).

19. Takeuchi, O. \& Akira, S. Pattern recognition receptors and inflammation. Cell 140 , 805-820 (2010).

20. Kawai, T. et al. IPS-1, an adaptor triggering Rig-I- and Mda5-mediated type I interferon induction. Nat. Immunol. 6, 981-988 (2005).

21. Meylan, E. et al. Cardif is an adaptor protein in the Rig-I antiviral pathway and is targeted by hepatitis C virus. Nature 437, 1167-1172 (2005).

22. Seth, R.B., Sun, L., Ea, C.K. \& Chen, Z.J. Identification and characterization of Mavs, a mitochondrial antiviral signaling protein that activates NF- $\mathrm{KB}$ and Irf 3 . Cell 122, 669-682 (2005).

23. Xu, L.G. et al. VISA is an adapter protein required for virus-triggered Ifn- $\beta$ signaling Mol. Cell 19, 727-740 (2005).

24. Gitlin, L. et al. Essential role of mda-5 in type I IFN responses to polyriboinosinic: polyribocytidylic acid and encephalomyocarditis picornavirus. Proc. Natl. Acad. Sci. USA 103, 8459-8464 (2006).

25. Kato, H. et al. Differential roles of Mda5 and Rig-I helicases in the recognition of RNA viruses. Nature 441, 101-105 (2006).

26. Yoneyama, M. et al. The RNA helicase Rig-I has an essential function in double-stranded RNA-induced innate antiviral responses. Nat. Immunol. 5, 730-737 (2004).

27. Sadler, A.J. \& Williams, B.R. Interferon-inducible antiviral effectors. Nat. Rev. Immunol. 8, 559-568 (2008).

28. Bogdan, C., Mattner, J. \& Schleicher, U. The role of type I interferons in non-viral infections. Immunol. Rev. 202, 33-48 (2004).

29. Dondorp, A.M. et al. Estimation of the total parasite biomass in acute falciparum malaria from plasma PfHRP2. PLoS Med. 2, e204 (2005).

30. Mohd Hanafiah, K., Groeger, J., Flaxman, A.D. \& Wiersma, S.T. Global epidemiology of hepatitis $C$ virus infection: new estimates of age-specific antibody to HCV seroprevalence. Hepatology 57, 1333-1342 (2013).

31. Roberts, Z.J. et al. The chemotherapeutic agent DMXAA potently and specifically activates the TBK1-Irf-3 signaling axis. J. Exp. Med. 204, 1559-1569 (2007).

32. Shirey, K.A. et al. The anti-tumor agent, 5,6-dimethylxanthenone-4-acetic acid (DMXAA), induces Ifn- $\beta$-mediated antiviral activity in vitro and in vivo. J. Leukoc. Biol. 89, 351-357 (2011).

33. González-Navajas, J.M., Lee, J., David, M. \& Raz, E. Immunomodulatory functions of type I interferons. Nat. Rev. Immunol. 12, 125-135 (2012).

34. Mourier, T. et al. Genome-wide discovery and verification of novel structured RNAs in Plasmodium falciparum. Genome Res. 18, 281-292 (2008).

35. Manel, N. et al. A cryptic sensor for HIV-1 activates antiviral innate immunity in dendritic cells. Nature 467, 214-217 (2010).

36. Andrade, B.B. et al. Hepatitis B infection is associated with asymptomatic malaria in the Brazilian Amazon. PLoS ONE 6, e19841 (2011).

37. Ouwe-Missi-Oukem-Boyer, O. et al. Hepatitis C virus infection may lead to slower emergence of P. falciparum in blood. PLOS ONE 6, e16034 (2011). 


\section{ONLINE METHODS}

Mice. Mice were housed in the facilities of the Institute of Molecular Medicine in Lisbon, the University of Massachusetts Medical School (Worcester) and the University Hospital of Bonn. C57BL/6J, CD-1 and BALB/c wild-type mice were purchased from Charles River Breeding Laboratories. Ifnarl-deficient mice were bred in specific pathogen-free facilities at the Instituto Gulbenkian de Ciência. Ifnar $1^{\text {flox/flox }}$ (ref. 38) were used to generate LysM-Cre-Ifnar $1^{\text {flox/flox }}$

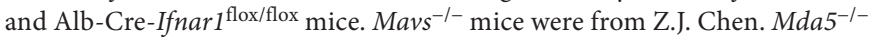

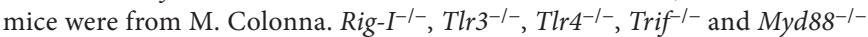
mice were a gift from S. Akira. Trif ${ }^{-1-}$ and $\mathrm{Myd} 88^{-/-}$mice were used for generating Trif ${ }^{-1-}$; $M y d 88^{-/-}$mice. Rig- $I^{-/-}$mice were kept on an outbred background (CD1 ICR). Irf $f 9^{-I-} / \operatorname{Irf} 7^{-/-}$and $I r f 3^{-/-}$mice were kindly provided by Tadatsugu Taniguchi, and Ifit1-lac $Z^{13}$ mice were provided by G.S.-F. Mice used in this manuscript are in the C57BL/6Jbackground and have been backcrossed at least ten times. Male mice were used. All in vivo protocols were approved by the internal animal care committee of the Instituto de Medicina Molecular and were performed according to national and European regulations.

Parasite strains, liver infection and blood parasitemia. GFP- or luciferaseexpressing $P$. berghei ANKA $(P b) \mathrm{WT}^{39,40}$ and $\mathrm{p} 36 \mathrm{p}$-deficient ${ }^{16}$ P. yoelii $17 \mathrm{XNL}$ $(P y)$ 17XNL and $P$. berghei NK65 WT, uis3- and uis4-deficient ${ }^{14,15}$ sporozoites were obtained by dissection of Anopheles stephensi-infected mosquitoes bred at the Institute of Molecular Medicine and New York University insectariums. For all experiments with P. berghei, the ANKA strain was used except for the experiment presented in Supplementary Figure 2, for which we used the NK65 strain. Mice were injected intravenously with the designated quantity of sporozoites. Mosquito bite infection was performed with ten mosquitoes per mouse except when specified otherwise. Parasite liver load was quantified by qRT-PCR in total liver of infected mice after indicated time points of infection. The presence of erythrocytic-stage parasites was monitored by Giemsa-stained blood smears and fluorescence activated cell sorting (FACS). For FACS measurements, using the FACS Calibur (BD Biosciences), a drop of blood in $1 \times$ PBS was used to measure the blood parasitemia. GFP-expressing parasites were detected in the green fluorescent channel FL1-H. The gated amount related to all detected live cells corresponds to the blood parasitemia in percent. FACS data were analyzed using FlowJo software (version 9.0.2, Tree Star Inc., Oregon, USA).

RNA isolation of total livers and QRT-PCR quantification. For mouse liver RNA extraction, whole livers were homogenized in $3 \mathrm{ml}$ denaturing solution (4 M guanidine thiocyanate; $25 \mathrm{mM}$ sodium citrate $\mathrm{pH} 7,0.5 \% \mathrm{~N}$-lauroylsarcosine and, $0.7 \% \beta$ mercaptoethanol in DEPC-treated water). RNA was extracted using RNeasy Mini kit (Qiagen). Complementary DNA was synthesized using Transcriptor First Strand cDNA Synthesis kit (Roche). Gene expression analysis was performed using kits from Applied Biosystems. For analysis, the expression levels of all target genes were normalized against hypoxanthine guanine phosphoribosyltransferase $(\mathrm{Hprt})$ housekeeping gene $(\Delta \mathrm{Ct})$. Gene expression values were then calculated based on the $\Delta \Delta \mathrm{Ct}$ method, using the mean of control group as calibrator to which all other samples were compared. Primer pairs used to detect target gene transcripts are listed in Supplementary Table 3.

Transcription profiling. The profiles of sporozoite-infected livers were previously published by Portugal et al. ${ }^{10}$. The original data from Portugal et al. ${ }^{10}$ and our new data in mice lacking Ifnar 1 were submitted to Array Express and are accessible with the accession code E-MTAB-1145. Arrays were normalized using the robust multiarray averaging (RMA) method $^{41}$ as implemented in the Bioconductor oligo package ${ }^{42}$, and differential expression was detected using linear models and empirical Bayesian methods as implemented in the Bioconductor limma package ${ }^{43}$. Three biological replicates were analyzed for each group. We found 1,088 genes were differentially expressed $(P<0.05)$ when the sporozoite-infected group was compared to the Sg-infected control. Of these, 86 were at least twofold up regulated. Gene ontology term overrepresentation was measured using the GOstats tool ${ }^{44}$.

RNA isolation from hepatocytes and nonparenchymal cells. Mouse primary hepatocytes were isolated using a modified two-step perfusion protocol followed by a Percoll purification step ${ }^{45,46}$. Briefly, mice were killed by $\mathrm{CO}_{2}$ inhalation and immediately processed for cannulation of the portal vein using a 26-gage needle. Upon successful cannulation, the inferior vena cava (IVC) was cut to allow fluid to drain. Liver perfusion medium (LPM, Gibco) was perfused at $8-9 \mathrm{~mL} / \mathrm{min}$ for $10 \mathrm{~min}$ followed by liver digestion medium (LDM, Gibco) also at a rate of 8-9 $\mathrm{mL} / \mathrm{min}$ for $10 \mathrm{~min}$. Intermittent clamping of the IVC (3-s clamp every $30 \mathrm{~s}$ ) was performed during LDM perfusion to improve tissue digestion. After digestion, the liver was excised and the cells were liberated by tearing and shaking of the liver with forceps. The cell suspension was then sequentially filtered through a $100-\mu \mathrm{m}$ and a $70-\mu \mathrm{m}$ cell strainer and spun at $50 \mathrm{~g}$ for $3 \mathrm{~min}$. The cell pellet was resuspended in Williams's Medium E (Gibco) with $10 \%$ of fetal calf serum (FBS, Gibco) and carefully overlaid on a $60 \%$ Percoll solution (1:1). The cell suspension was fractionated by centrifugation at $750 \mathrm{~g}$ for $20 \mathrm{~min}$, without break, at $20^{\circ} \mathrm{C}$. Viable hepatocytes deposited in the pellet were washed with Williams's Medium E with $10 \%$ FBS, spun at $50 \mathrm{~g}$ for 3 min and resuspended in complete Williams's Medium E (supplemented with $4 \% \mathrm{FBS}$ and $1 \%$ penicillin/streptomycin, Gibco). Viability and yield were assessed by trypan blue staining. To obtain mouse primary nonparenchymal cells, perfused livers were squeezed in $1 \times$ PBS solution containing DNase $\left(2 \mathrm{U} \mathrm{ml}^{-1}\right)$, filtered through a $70-\mu \mathrm{m}$ filter followed by 8 -min centrifugation at 1,300 r.p.m. The pellet was resuspended in $10 \mathrm{ml}$ of $35 \%$ Percoll and centrifuged at 2,600 r.p.m. for $20 \mathrm{~min}$ without break at $20^{\circ} \mathrm{C}$. The pellet representing the nonparenchymal cells was washed in $1 \times \mathrm{PBS}$ containing $2 \%$ serum before resuspension into lysis/binding buffer. qRT-PCR analysis of the relative expression of macrophage (Cd68), neutrophil (Ncf2) and hepatocyte (Apoa1) markers was performed to confirm purity of the obtained populations.

Irf3 bone marrow-chimeric mice. Chimeric mice were generated by transplanting WT (C57BL/6) bone marrow (BM) into lethally $\gamma$-irradiated (900 rads), Irf3deficient mice (Irf3 ${ }^{-/-}$WT BM) or by transplanting Irf3-deficient bone marrow into irradiated, WT mice (WT Irf3 $3^{-1-} \mathrm{BM}$ ). Bone marrow cells from donor mice $\left(1 \times 10^{7}\right.$ cells $)$ were injected into each recipient mouse through the retro-orbital venous plexus $1 \mathrm{~d}$ after irradiation. Chimeric animals were allowed to recover for 8 weeks before sporozoite infection was initiated. Depletion of circulating cells and reconstitution with donor cells was confirmed in all chimeric mice by PCR of blood genomic DNA. Blood DNA was extracted using QIAamp DNA Micro Kit (Qiagen). PCR was performed using PCR Master Mix (Promega kit) and a set of three primers that amplify sequence either from the WT (374 bp) or knockout (500 bp) allele. The following oligonucleotides $\left(5^{\prime}-3^{\prime}\right)$ were used for specific amplification: GAACCTCGGAGTTATCCCGAAGG, GTTTGAGTTATCCCTGCACTTGGG and TCGTGCTTTACGCTATCGCCGCTCCCGATT. Amplification program: $95^{\circ} 15$ min 1 cycle; $95^{\circ} 30 \mathrm{~s}, 60^{\circ} 1 \mathrm{~min}, 72^{\circ} 2 \min 35$ cycles; $4^{\circ} \mathrm{C}$ hold.

Western blot analysis. Livers were lysed in RIPA buffer ( $50 \mathrm{mM}$ Tris- $\mathrm{HCl} \mathrm{pH}$ 7.4, $150 \mathrm{mM} \mathrm{NaCl}, 0,25 \% \mathrm{Na}$-deoxycholate and a protease inhibitor cocktail). $20 \mu \mathrm{g}$ of total lysates were resolved on $10 \%$ SDS-PAGE and transferred to a nitrocellulose membrane (Hybond-ECL, GE Healthcare). IFIT1- and DAIspecific bands were detected by using the primary antibodies (mouse antimouse IFIT1 $(1: 5,000)$ and rabbit anti- mouse DAI $(1: 2,000)$, kindly provided by G.S.-F.). Mouse anti-mouse $\beta$-tubulin (1:2,000) (Santa Cruz sc-58886) was used as a loading control. Horseradish peroxidase (HRP)-conjugated anti-mouse and anti-rabbit IgG F( $\left.\mathrm{ab}^{\prime}\right) 2$ fragment (GE Healthcare) were used as secondary antibodies. The membrane was developed using the ECL Western Blotting Analysis System, according to the manufacturer's instructions (GE Healthcare).

Bone marrow-derived dendritic cell generation. Bone marrow was isolated from femora and tibiae of 8 -week-old mice. Bone marrow was cultured in RPMI 1640 supplemented with L-glutamine, sodium pyruvate, nonessential amino acids, $50 \mu \mathrm{g} \mathrm{ml}^{-1}$ penicillin/streptomycin (Invitrogen) and granulocytemacrophage colony-stimulating factor (3\%) to generate dendritic cells. After $7 \mathrm{~d}$, cells were plated in 96 -well plates at a density of $2 \times 10^{5}$ cells per well and used for subsequent experiments.

Plasmodial and viral RNAs. $P$. berghei- and $P$. yoelii-infected blood samples were purified using a CF11 cellulose column ${ }^{47}$. P. falciparum $3 \mathrm{D} 7$ was grown in recently collected donor erythrocytes in RPMI-based complete malaria culture medium (CMCM) according to the recommendations of the Malaria Research and Reference Reagent Resource Center (MR4). Plasmodium RNAs were then 
isolated using Trizol from infected erythrocytes according to manufacturer's instructions. Briefly, RIG-I ligand 3pRNA was an in vitro-transcribed, bluntended ppp-dsRNA 48-mer based on the GFP2 sequence ${ }^{48}$. For HCV RNA synthesis p90/HCVFL-long pU, an infectious HCV cDNA clone consisting of the consensus cDNA of HCV genotype la isolate H77 (ref. 49) was used to create a high-copy-number derivative in the pUC19 vector backbone (pUC/H77FL-X). $\mathrm{pUC} / \mathrm{H} 77 \mathrm{FL}-\mathrm{X}$ includes a $5^{\prime} \mathrm{T} 7$ promoter and a unique $\mathrm{XbaI}$ runoff site for production of full-length RNA transcripts. A replication-defective derivative (pUC/ H77FL-X pol-) was created by mutating polymerase active-site residues GDD to AGG. Transcript RNA was synthesized by in vitro transcription as described previously ${ }^{50}$. Briefly, plasmid DNA was linearized with XbaI and purified with the MinElute PCR purification kit (Qiagen Sciences, MD). Linearized template DNA was transcribed using the T7 RiboMAX Express large-scale RNA production system (Promega, WI) according to the manufacturer's recommendations. Transcript RNA was treated with DNase I and purified using an RNeasy mini kit (Qiagen Sciences). RNA integrity was confirmed by agarose gel electrophoresis.

RNA quality control. RNAs ( $1 \mu \mathrm{g}$ per lane) were resuspended in RNA loading buffer (Fermentas) and denatured for $5 \mathrm{~min}$ at $65^{\circ} \mathrm{C}$, then chilled on ice. Samples were run on a standard TAE (0.8\%) gel (Ultrapure Agarose from Invitrogen, RNAse-free TAE from Carl-Roth) at $5 \mathrm{~V} \mathrm{~cm}^{-1}$ for $1 \mathrm{~h}$. The gel was subsequently stained with ethidium bromide and visualized by ultraviolet transillumination. Size standards were from Fermentas (Generuler DNA Ladder Mix, 100 bp-10 kb and RibuRuler High Range RNA ladder 0.2-6 kb).

Stimulation of dendritic cells and hepatocytes. 3pRNA, poly(I:C) and total $P$. falciparum, $P$. berghei and $P$. yoelii RNA samples were transfected with Lipofectamine 2000 (Invitrogen) according to the manufacturer's instructions. Briefly, $200 \mathrm{ng}$ RNA were complexed to $0.6 \mu \mathrm{l}$ transfection reagent in $50 \mu \mathrm{l}$ of Opti-MEM. Following $20 \mathrm{~min}$ incubation at room temperature (RT), complexes were added to cells. Supernatants were collected $18 \mathrm{~h}$ after transfection.

Mouse IFN- $\boldsymbol{\alpha}$ and IFN- $\beta$ ELISA. IFN- $\alpha$ and IFN- $\beta$ levels were determined using a sandwich ELISA. Rat monoclonal antibody to mouse IFN- $\alpha$ and IFN- $\beta$ (PBL Biomedical Laboratories clone RMMA- 1 for IFN- $\alpha$ and clone RMMB- 1 for IFN- $\beta$ ) was used as capture antibody ( $2 \mu \mathrm{g}$ per $\mathrm{ml}$ for coating), rabbit polyclonal antibody to mouse IFN- $\alpha$ and IFN- $\beta$ (PBL Biomedical Laboratories 32100-1 for IFN- $\alpha$ and 32400-1 for IFN- $\beta$ ) was used at 80 neutralizing units per $\mathrm{ml}$ for detection and HRP-conjugated donkey anti-rabbit IgG $(1: 3,000)$ (Bio-Rad 170-6515) was used as secondary reagent. Recombinant mouse IFN- $\alpha$ and IFN- $\beta$ (PBL Biomedical Laboratories, $12100-1$ for IFN- $\alpha$ and $12400-1$ for IFN- $\beta$ ) were used as standards.

In vivo injection of RNAs in mice. RNAs were hydrodynamically transfected intravenously with naked RNA.

Immunohistochemical staining of liver sections. Livers were fixed with $4 \%$ PFA at RT for $2 \mathrm{~h}$. The fixed liver lobes were cut into $40-\mu \mathrm{m}$-thick sections using the Vibratome VT 1000 S (Leica). Liver sections were blocked in 2\% BSA and $0,3 \%$ Triton for $4{ }^{\circ} \mathrm{C}$ overnight. The following primary antibodies were used to stain liver sections: rabbit anti-GFP polyclonal antibody conjugated to Alexa Fluor 488 (1:400) (Invitrogen) to stain for GFP-expressing liver-stage parasites; chicken anti-lacZ polyclonal antibody $(1: 1,000)$ (Abcam, ab9361) to stain for $\beta$ - galactosidase, rabbit polyclonal anti-MSP-1 antibody (1:300) (kindly provided by A. Holder); goat anti-P. berghei UIS4 $(1: 1,000)^{51}$, kindly provided by M. Seabra, and mouse anti- $P$. berghei HSP70 $(2 \mathrm{E} 6,1: 1,000)^{52}$. Incubations were performed at room temperature for about $6 \mathrm{~h}$ or at $4{ }^{\circ} \mathrm{C}$ overnight. The following fluorescently tagged secondary antibodies were used for detection: donkey anti-chicken antibody conjugated to Alexa Fluor 594, donkey anti-mouse conjugated to Alexa Fluor 488 and donkey anti-goat conjugated to Alexa Fluor 488 (all 1:500 from Jackson ImmunoResearch Laboratories: 703-516-155, 715546-150 and 705-485-003, respectively) and donkey anti-rabbit antibody conjugated to Alexa Fluor 546 (1:500, Life Technologies, A10040). Cell nuclei were stained with DAPI. Stained liver sections were placed on microscope slides and mounted with $24 \times 60 \mathrm{~mm}$ cover slips using Fluoromount-G (SouthernBiotech). All images were acquired on LSM 510 META confocal laser point-scanning microscope (Zeiss).
Definition of infiltrates for scoring. Intrahepatic parasites were defined to be associated with infiltrates when the infected cells were in contact with more than three immune cells. Immune cells were identified by DAPI staining, based on their cytologic aspect (size of the cell and nucleus:cytoplasmic ratio).

Quantification of $\beta$-galactosidase expression in liver sections of Ifit1-lacZ reporter mice. Images were acquired using a Zeiss LSM 510 confocal microscope and a $40 \times$ water objective. Imaging settings that would prevent saturated pixels were determined on the infected slices (where the IFIT-lacZ signal was strongest). Identical settings were subsequently used for all images. 15 parasites (equidistant from the top of the slice, based on DAPI intensity from hepatocyte nuclei) were imaged in each liver taken per mouse. In the uninfected controls, 15 images of identically-sized fields of view randomly distributed throughout the specimens were acquired. Two mice were used for each condition, so 30 images were analyzed for both conditions. The mean fluorescence intensity (MFI) of the $\beta$-galactosidase staining for each image was determined. The mean value of MFI of the pictures of infected samples was compared to the mean MFI of noninfected samples and given as respective fold induction. Image analysis was performed in the LSM Image Browser (Zeiss) with Fiji image processing package.

Blood transfer experiments. C57BL/6J mice were injected intravenously with $5 \times 10^{4}$ GFP-expressing P. berghei sporozoites. At $42 \mathrm{~h}$ and $48 \mathrm{~h}$ after infection, the mice were bled by cardiac puncture using heparin $\left(100 \mathrm{U} \mathrm{ml}^{-1}\right)$ as an anticoagulant. Approximately $0.8 \mathrm{ml}$ of blood was obtained from each mouse. One-fourth (corresponding to about $200 \mu \mathrm{l}$ ) of the recovered blood was injected intravenously into each recipient mouse. Recipients were monitored for the presence of erythrocytic-stage parasites by Giemsa-stained blood smears and FACS to determine the percentage of infected red blood cells until day 12 after blood inoculation.

Statistical Analysis. Data are expressed as means \pm s.e.m. Statistically significant differences between two different groups were analyzed using the Mann-Whitney test except when specified otherwise. $P<0.05$ were considered statistically significant, except when specified otherwise. Significances are represented in the figures as follows ${ }^{\star} P<0.05 ;{ }^{* \star} P<0.01 ;{ }^{* \star} P<0.001$. All statistic tests were performed using Graph Prism 5.0 software.

38. Kamphuis, E., Junt, T., Waibler, Z., Forster, R. \& Kalinke, U. Type I interferons directly regulate lymphocyte recirculation and cause transient blood lymphopenia. Blood 108, 3253-3261 (2006).

39. Franke-Fayard, B. et al. A Plasmodium berghei reference line that constitutively expresses GFP at a high level throughout the complete life cycle. Mol. Biochem. Parasitol. 137, 23-33 (2004).

40. Ploemen, I.H. et al. Visualisation and quantitative analysis of the rodent malaria liver stage by real time imaging. PLOS ONE 4, e7881 (2009).

41. Irizarry, R.A. et al. Exploration, normalization, and summaries of high density oligonucleotide array probe level data. Biostatistics 4, 249-264 (2003).

42. Carvalho, B.S. \& Irizarry, R.A. A framework for oligonucleotide microarray preprocessing. Bioinformatics 26, 2363-2367 (2010).

43. Gentleman, R. et al. limma: Linear Models for Microarray Data. in Bioinformatics and Computational Biology Solutions using R and Bioconductor 397-420 (Springer New York, 2005).

44. Falcon, S. \& Gentleman, R. Using GOstats to test gene lists for GO term association. Bioinformatics 23, 257-258 (2007).

45. Gonçalves, L.A., Vigario, A.M. \& Penha-Goncalves, C. Improved isolation of murine hepatocytes for in vitro malaria liver stage studies. Malar. J. 6, 169 (2007).

46. Zhang, W. et al. PCB 126 and other dioxin-like PCBs specifically suppress hepatic PEPCK expression via the aryl hydrocarbon receptor. PLOS ONE 7, e37103 (2012).

47. Sriprawat, K. et al. Effective and cheap removal of leukocytes and platelets from Plasmodium vivax infected blood. Malar. J. 8, 115 (2009).

48. Schlee, M. et al. Recognition of $5^{\prime}$ triphosphate by RIG-I helicase requires short blunt double-stranded RNA as contained in panhandle of negative-strand virus. Immunity 31, 25-34 (2009).

49. Kolykhalov, A.A. et al. Transmission of hepatitis $C$ by intrahepatic inoculation with transcribed RNA. Science 277, 570-574 (1997).

50. Saeed, M. et al. Efficient replication of genotype $3 a$ and $4 a$ hepatitis C virus replicons in human hepatoma cells. Antimicrob. Agents Chemother. 56, 5365-5373 (2012).

51. Lopes da Silva, M. et al. The host endocytic pathway is essential for Plasmodium berghei late liver stage development. Traffic 13, 1351-1363 (2012).

52. Tsuji, M., Mattei, D., Nussenzweig, R.S., Eichinger, D. \& Zavala, F. Demonstration of heat-shock protein 70 in the sporozoite stage of malaria parasites. Parasitol. Res. 80, 16-21 (1994). 